\title{
Lysosomal Storage Disorders: Present and Future
}

\author{
Shubha R PhadKe \\ Department of Medical Genetics, Sanjay Gandhi Postgraduate Institute of Medical Sciences, Lucknow, India. \\ shubharaophadke@gmail.com
}

$\mathrm{L}$ ysosomal storage disorders (LSDs) is a group of more than 50 single gene disorders caused by deficiencies of any of the glycoprotein acid hydrolase enzymes. Lysosomes are intracellular organelles serving important functions of breakdown of large molecules and helping in their disposal. In case of deficiency of any of these enzymes, there is deposition of large amounts of the substrate compound inside the lysosomes, leading to hepatosplenomegaly along with variable involvement of brain, bones, connective tissue and heart. Mucopolysaccharidosis (MPS) is a prototype of LSDs, and has been an easy diagnosis in pediatric practice. Patients presenting with coarse facies, hepatosplenomegaly, short stature, joint contractures, radiological changes of dysostosis multiplex, with or without corneal clouding, are clinically labeled as mucopolysaccharidosis. It is important to confirm the subtypes of such cases by enzyme assays and mutation detection, both of the diagnostic tests being available in India. It is important to note that mucolipidosis and oligosaccharidosis have clinical features similar to MPS, and need to be considered when urine test result is negative or there is another clinical clue. Other than classic MPS phenotype, LSDs may present with only neurological manifestations during infancy, childhood or even adulthood. Metachromatic leukodystrophy, Krabbe disease and Gangliosidosis are examples of such diseases. Cholestatic jaundice, cardiac failure and fetal hydrops are other presenting features of LSDs. Fabry disease needs a special mention as its diagnosis is often delayed for decades due to vague symptoms of neuralgia and absence of specific clinical signs. Presence of acroparesthesias with or without angiokeratomas suggests the diagnosis. This disorder is clinically different from other LSDs, and is a cause of cardiomyopathy, stroke and end-stage renal disease in young adults. Fabry disease is an X-linked disease, but manifestations in females are also observed commonly.

Suspecting LSDs in appropriate clinical situations is necessary as definitive therapy in the form of enzyme replacement therapy (ERT) is available for MPS I (Hurler syndrome), MPS II (Hunter syndrome), MPS VI (Maroteaux Lammy syndrome) and Fabry disease [1]. Cerazyme, an ERT for non-neuropathic Gaucher disease was first such agent available for patient care since 1996. Indian experience also shows significant improvement in signs and symptoms of the disease and hematological parameters within a short period of therapy of six months [2]. Other ERTs have also been shown to be effective [3], but the variability of efficacy of ERT is a major issue. Other than development of antibodies and its effect on the efficacy of ERT and immunological reaction to ERT, inability to deliver the enzyme to brain and bones is the most important challenge for ERT. Other form of therapy for LSDs is reduction of substrate. One such molecule, Miglustat, is found to be effective as a maintenance therapy after improvement with ERT in Gaucher disease [4], but is not much effective in improving neurological manifestations of Niemann Pick disease [5]. Presence of neuronopathic variants of all LSDs is common as illustrated by a large series of LSDs presenting as neuroregression published in this issue of Indian Pediatrics [6]. Success of ERTs in some of the LSDs has brought rays of hope for other LSDs with poor outcome. Bone marrow transplantation has been tried with variable outcome in LSDs [7]. Presymptomatic hematopoetic stem cell transplantation (HSCT) may be effective, and hence newborn screening for Krabbe disease has been proposed. Although early treatment with HSCT seems to alter early-childhood mortality and some of the morbidity associated with early-infantile Krabbe disease, a lot more understanding about the disease course and efficacy of such novel strategy is needed before including LSDs in newborn screening program [8]. Newborn screening for Pompe disease and early institution of ERT within a few weeks of birth has shown significantly improved outcome, but inspite of achieving independent walking, many children developed hip girdle muscle weekness, ptosis and speech delays [9]. Development of antibodies is an important hindrance in the efficacy of ERT for Pompe disease as was seen in Indian patients (Personal communications). Delay in the diagnosis causing delay in starting ERT is another cause of unsatisfactory outcome 
in Pompe disease. The challenges ahead are enormous. Targeting enzyme to lysosome, the first critical step has been achieved. Developing an enzyme that reaches brain and bones, and prevents and improves all manifestations of LSDs, will cause dramatic change in the outcome. The strategies to overcome blood-brain barrier, including stimulation of other receptors, and gene therapy are being explored $[10,11]$.

The developments in the field of LSDs are being reflected in the Indian scenario $[12,13]$. Though individually rare, prevalence of LSDs is 1 per 7- to 8 thousand worldwide. For a larger population in India, the absolute number of cases obviously must be larger. However, the cases diagnosed in India at present represent the tip of an iceberg. Presence of high level of consanguinity in India is obvious in LSDs, and account for about one-third of cases [11]. Confirmation of diagnosis by enzyme assays and mutation detection is important not only for diseases for which ERT is available, but also for genetic counseling and prenatal diagnosis as all these disorders are associated with $25 \%$ risk of recurrence in the siblings [14,15]. It is important to diagnose mild or attenuated and juvenile/adult onset variants of LSDs, and offer them ERT if available. Screening of relatives of a confirmed case will identify more cases with subclininical manifestations. The cost of ERTs is exorbitant, and is beyond reach of most patients. Some of the drug companies are providing ERT to limited number of patients under their humanitarian access programs. Combined efforts of patient support groups, clinicians, scientific community and policy makers are needed to look for long-term solutions to provide therapy to sizable numbers of the treatable LSDs. Supportive therapies, surveillance for early diagnosis of complications, and genetic counseling are important components of management. Awareness about various presentations of LSDs, approach to diagnosis, use of diagnostic facilities, and genetic counseling will go a long way in helping the patients and families with this rare group of genetic disorders.

Acknowledgement: Indian Council of Medical Research for establishing National Task Force and funding multicentric project on lysosomal storage disorders.

Funding: None; Competing interests: None stated.

\section{REFERENCES}

1. Ratko TA, Marbella A, Godfrey S, Aronson N. EnzymeReplacement Therapies for Lysosomal Storage Diseases Technical Brief. No. 12. (Prepared by Blue Cross and Blue Shield Association Technology Evaluation Center Evidence-based Practice Center under Contract No. 2902007-10058-I.) Rockville, MD: Agency for Healthcare
Research and Quality. January 2013. Available from: www.effectivehealthcare.ahrq.gov/reports/final.cfm. Accessed November 08, 2015.

2. Nagral A, Mewawalla $P$, Jagadeesh S, Kabra M, Phadke SR, Verma IC, et al. Recombinant macrophage targeted enzyme replacement therapy for Gaucher disease in India. Indian Pediatr. 2011;48:779-84.

3. Tomanin R, Zanetti A, D'Avanzo F, Rampazzo A, Gasparotto N, Parini R, et al. Clinical efficacy of enzyme replacement therapy in paediatric Hunter patients, an independent study of 3.5 years. Orphanet J Rare Dis. 2014;9:129.

4. Shemesh E, Deroma L, Bembi B, Deegan P, Hollak C, Weinreb NJ, et al. Enzyme replacement and substrate reduction therapy for Gaucher disease. Cochrane Database Syst Rev. 2015;3:CD010324.

5. Di Rocco M, Barone R, Madeo A, Fiumara A. Miglustat does not prevent neurological involvement in Niemann Pick C disease. Pediatr Neurol. 2015;53:e15.

6. Sheth J, Mistri M, Bhavsar R, Sheth F, Kamate M, Shah H, et al. Lysosomal storage disorders in Indian children with neuroregression attending a genetic center. Indian Pediatr. 2015;52:1029-33.

7. Guffon N, Bertrand Y, Forest I, Fouilhoux A, Froissart R. Bone marrow transplantation in children with Hunter syndrome: Outcome after 7 to 17 years. J Pediatr. 2009;154:733-7.

8. Kemper AR, Knapp AA, Green NS, Comeau AM, Metterville DR, Perrin JM. Weighing the evidence for newborn screening for early-infantile Krabbe disease. Genet Med. 2010;12:539-43.

9. Chien YH, Lee NC, Chen CA, Tsai FJ, Tsai WH, Shieh JY, et al. Long-term prognosis of patients with infantile-onset Pompe disease diagnosed by newborn screening and treated since birth. J Pediatr. 2015;166: 985-91.

10. Urayama A, Dohgu S, Robinson SM, Sly WS, Grubb JH, Banks WA. Alpha adrenergic induction of transport of lysosomal enzyme across the blood-brain barrier. PLoS One. 2015;10:e0142347.

11. Biffi A, Montini E, Lorioli L, Cesani M, Fumagalli F, Plati $\mathrm{T}$, et al. Lentiviral hematopoietic stem cell gene therapy benefits metachromatic leukodystrophy. Science. 2013; 341:1233158.

12. Verma PK, Ranganath P, Dalal AB, Phadke SR. Spectrum of lysosomal storage disorders at a medical genetics center in Northern India. Indian Pediatr. 2012;49:799-804.

13. Phadke SR, Mandal K, Girisha KM. Fabry disease: A treatable lysosomal storage disorder. Natl Med J India. 2009;22:20-2.

14. Shukla P, Vasisht S, Srivastava R, Gupta N, Ghosh M, Kumar $\mathrm{M}$, et al. Molecular and structural analysis of metachromatic leukodystrophy patients in Indian population. J Neurol Sci. 2011;301:38-45.

15. Bidchol AM, Dalal A, Shah H, S S, Nampoothiri S, Kabra $\mathrm{M}$, et al. GALNS mutations in Indian patients with mucopolysaccharidosis IVA. Am J Med Genet A. 2014;164A(11):2793-801. 